\title{
Basic Foundations of Clinical Electrophysiology. Electrophysiological Mechanism of Phase 4 Conduction Block in The His Purkinje System
}

\section{A Comparison With Phase 3 Block}

\author{
Andrew L. Wit ${ }^{1 *}$, Mark E. Josephson ${ }^{2}$ \\ ${ }^{1}$ Department of Pharmacology, Columbia University College of Physicians and Surgeons, New York, New York, USA \\ ${ }^{2}$ Harvard-Thorndike Electrophysiology Institute, Cardiovascular Division, Department of Medicine, Beth Israel Deaconess Medical \\ Center, Harvard Medical School, Boston, Massachusetts, USA
}

\begin{abstract}
We describe the mechanism underlying paroxysmal AV block that follows a sudden long cycle length. The phases and characteristics of the Purkinje fiber action potential are reviewed. Phase 4 block occurs when an impulse conducts into regions of Purkinje cells that have undergone spontaneous (phase 4) depolarization causing a reduction in the level of the membrane potential during diastole. This results in a decrease in inward sodium current during the depolarization phase ( 0 ) of the action potential, slow conduction and conduction block. The mechanisms involved are discussed in detail and compared to phase 3 block.

Copyright @ 2015 Science International Corp.
\end{abstract}

\section{Key Words}

Conduction - Bundle branch block - A-V block Action potentials

\section{Introduction}

The case report by Mohammad Shenasa, M.D., Paroxysmal Atrioventricular Block, in this issue of the journal [1] describes a patient in whom a premature atrial or junctional depolarization is followed by a period of complete AV block. This ECG characteristic is termed, "Paroxysmal Atrioventricular Block," which has been defined by Lee et al. [2] as "a sudden, pause-depen-

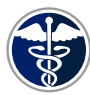

Fax +1 2037853346

E-Mail: agr@scienceinternational.org

http://arrhythmiagr.com/
๑) 2015 Arrhythmia Grand Rounds

Published by Science International Corp. ISSN 2326-4012 http://arrhythmiagr.com/
Accessible online at: dent phase 4 atrioventricular (A-V) block occurring in diseased conduction system often initiated by premature beats." Although no established predictors for paroxysmal A-V block exist, evidence of distal conduction system disease at baseline is usually present with a prolonged $\mathrm{H}-\mathrm{V}$ interval in the His bundle electrogram. A-V block occurs in the His Purkinje system, either in the His bundle or a bundle branches, following a prolongation of the cycle length of activation (prolonged $\mathrm{H}-\mathrm{H}$ interval) from spontaneous sinus rate slowing, post-extrasystolic pauses in association with atrial, ventricular and His extrasystoles, or supraventricular tachycardia . Interventricular conduction delay is present in the patient described by Shenasa [1].

Phase 4 block can also occur in the bundle branches to cause bundle branch block. Figure 1 shows this phenomenon. In Figure $1 \mathrm{~A}$ during atrial pacing at a cycle length of 800 msec with 1:1 A-V conduction, there is normal intraventricular conduction. In Figure $1 \mathrm{~B}$, atrial pacing at a cycle of $545 \mathrm{msec}$ results in 2:1 $\mathrm{A}-\mathrm{V}$ block in the $\mathrm{A}-\mathrm{V}$ node. As a result, the His Purkinje system is activated at a long cycle length of 1090 msec resulting in a wide QRS complex with a LBBB configuration.

Why and how does block occur after a long cycle? Paroxysmal AV block and bundle branch block after a long cycle length have been attributed to phase 4 block [1, 2]. What does that term mean and how is it

\footnotetext{
* Corresponding Author:

Andrew L. Wit

Department of Pharmacology

Columbia University College of Physicians and Surgeons

630 W168 St, New York, New York 10032, USA

Email: alw4@cumc.columbia.edu
} 


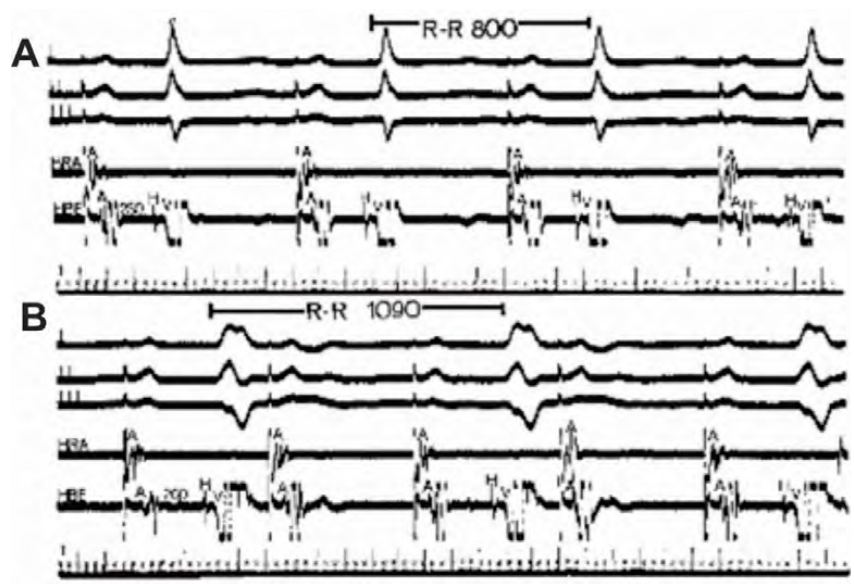

Figure 1. ECG (leads I, II, and III), and electrograms from high right atrium (HRA) and His bundle (HBE) during atrial pacing at a cycle length of $800 \mathrm{msec}(\mathbf{A})$ with 1:1 AV conduction and at a cycle length of $545 \mathrm{msec}$ (B) with 2:1 AV block.

different from phase 3 block?

\section{What Are the Phases of the Action Potentials in the His Purkinje System?}

Figure 2 shows diagrams of transmembrane action potentials recorded from Purkinje cells (myocytes) that form the His Purkinje conducting system. The action potential is divided into "phases". Phase 0 is depolarization from the negative level of the resting potential to the peak positive level (arrows). Phases 1, 2 , and 3 represent the different periods of repolarization back to the resting negative level. Phase 4 designates the membrane potential during diastole (arrows) which can either be at a steady negative level (Figure 2A) or can be spontaneously depolarizing when pacemaker activity is present (Figure 2B). Accordingly, phase 4 conduction block results when an impulse conducts into the His Purkinje system when myocytes are in the Phase 4 stage of their action potentials. This is to be contrasted with phase 3 conduction block when an impulse conducts into the His Purkinje system during phase 3 of repolarization.

\section{What Are the lonic Events during the Phases of the Action Potential?}

The ionic events during phase 0 are important de-

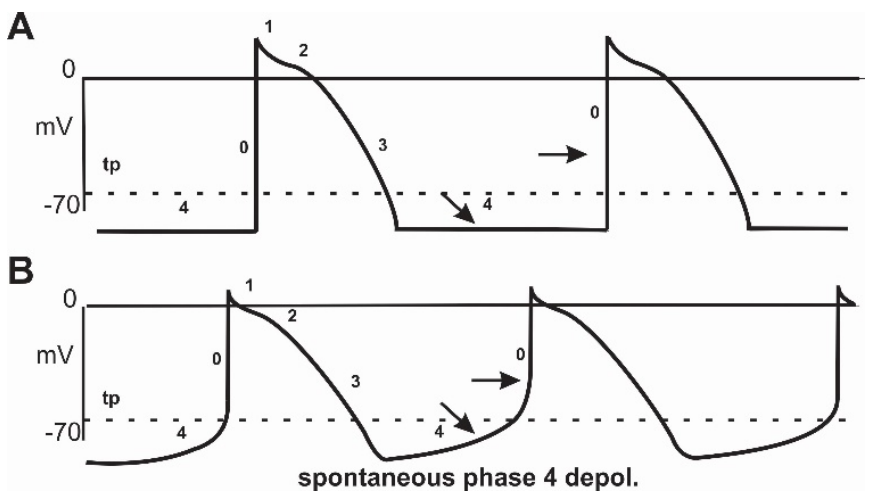

Figure 2. Diagrams of transmembrane action potentials characteristic of Purkinje myocytes. In each panel the horizontal black line indicates " 0 " potential. The phases of the action potentials are labeled 0-4. Note that in (A), diastolic (phase 4) potential has a steady negative value (arrow) while in (B), diastolic potential decreases spontaneously during phase 4 to a threshold potential (tp, dashed line) where phase 0 (arrow) is initialed.

terminants of the conduction properties in the His Purkinje system. Depolarization (movement of the membrane potential from the negative level of phase 4 to a positive level) during phase 0 is caused by the opening of channels in the sarcolemma that allows a rush of positive charges carried by $\mathrm{Na}^{+}$ions into the cell down a concentration gradient since there is a high concentration of $\mathrm{Na}^{+}$outside the cell and low concentration inside the cell.

$\mathrm{Na}^{+}$channels are formed by activation and inactivation "gates" diagrammed in Figure 3A, m represents the activation gate and $h$ the inactivation gate as originally named by Hodgkin and Huxley in their studies on the squid axon [3]. The "gates" are proteins in the sarcolemma that can move to open and close the channels. During the highly negative normal resting potential of phase 4 (around $-90 \mathrm{mV}$ ) the channels are closed by the $m$ gate while the $h$ gates are in an open state (Figure 3A, rest). Channels are stimulated to open by an electrical pulse, either from a conducting impulse or an external stimulus (m gate opens). $\mathrm{Na}^{+}$rushes into the cell as the $\mathrm{Na}^{+}$current $\left(\mathrm{I}_{\mathrm{Na}+}\right)$ during phase 0 (Figure $3 \mathrm{~A}$, activated). As the membrane potential moves in the positive direction, the $\mathrm{Na}^{+}$ channels begin to close, called $\mathrm{h}$ gate inactivation, reducing and eventually terminating $\mathrm{I}_{\mathrm{Na}+}$ (Figure $3 \mathrm{~A}$, inactivated). The inactivation is voltage dependent since it is caused by the decreasing membrane po- 


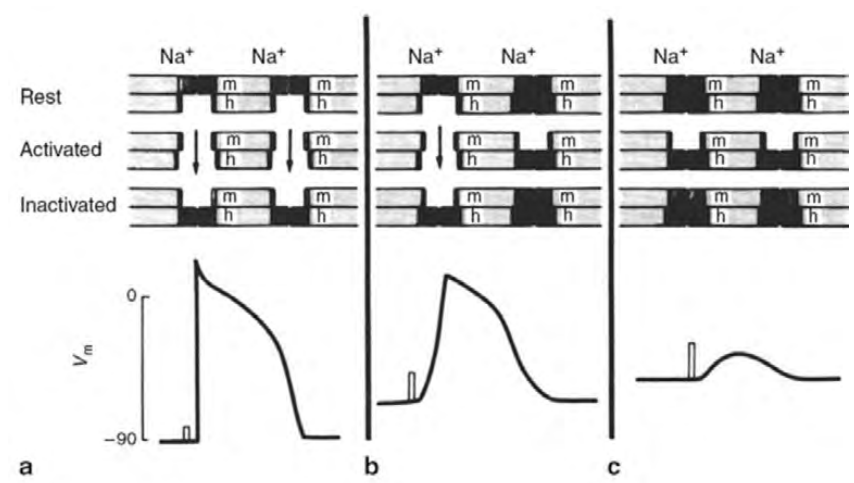

Figure 3. Diagram of the effects of decrease in membrane potential on the depolarization phase (0) of working myocardial cells. A diagram of the $\mathrm{Na}+$ channels are above action potentials. $\mathrm{Na}+$ channels have $\mathrm{m}$ and $\mathrm{h}$ gates which (top left) are closed in the resting state, open at high levels of membrane potential (activated) to allow inward $\mathrm{Na}+$ current and then close as repolarization inactivates them. Middle and right show increasing number of channels do not open when membrane potential is reduced to less negative values.

tential as the cell depolarizes during phase 0 . From here, the process of repolarization (phases 1, 2, and 3) returns the membrane potential to the negative resting membrane potential. Repolarization is to a large extent a result of positive charges carried by $\mathrm{K}^{+}$ currents $\left(I_{K_{+}}\right)$flowing out of the cell through special $\mathrm{K}^{+}$channels that open when the cell is depolarized. The myocyte exists in a refractory state, referred to as the effective refractory period, and cannot be excited again until phase 3 repolarization restores membrane potential to about two thirds of its original value. The time during the latter period of phase 3 is the relative refractory period where stimulation of myocytes causes a reduced, slowly conducting response.

\section{What Is the Relationship of the $\mathrm{Na}^{+}$Current $\left(\mathrm{I}_{\mathrm{Na}+}\right)$ to Conduction Block?}

The magnitude and intensity of the inward membrane current carried by $\mathrm{Na}^{+}\left(\mathrm{I}_{\mathrm{Na}+}\right)$ during phase 0 determines the speed of conduction of the cardiac impulse. The magnitude of the current (phase 0) in turn, depends on the level of the membrane potential during phase 4 at which it is initiated; it is voltage dependent.

Figure $4 \mathrm{~A}$ shows three myocytes representing

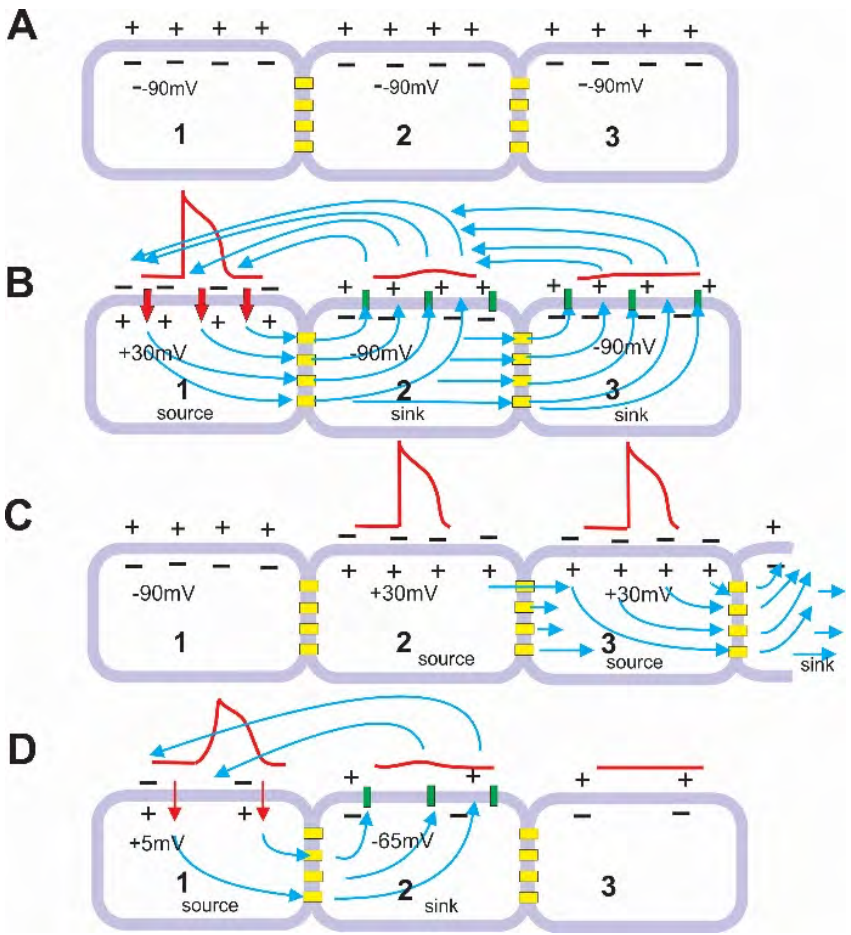

Figure 4. Diagram of conduction in a linear strand of three cells (rectangles 1-3) connected by gap junctional channels (yellow). Distribution of positive $(+)$ and negative $(-)$ charges across sarcolemma is shown as is the intracellular membrane potential. Membrane potentials generated by each cell is indicated above in red. Extracellular and intracellular current flow is shown by blue arrows.

a small segment of the His Purkinje system, at rest (during phase 4) with inside $-90 \mathrm{mV}$ negative to the outside, within the normal range. In Figure 4B, myocyte $A$ is excited and inward $I_{\mathrm{Na}+}$ (red arrows) generates an action potential (above the cell in red), with the inside of the cell becoming $+30 \mathrm{mV}$ with respect to the outside (also within the normal range). The juxtaposition of the excited myocyte (1) and the adjacent unexcited myocytes ( 2 and 3 ) results in flow of positive charges intracellularly, called axial current, between excited (myocyte 1 ) and unexcited myocytes ( 2 and 3 ) (from positive to negative) through the gap junction connections (yellow) which are intracellular channels connecting myocytes, indicated by the curved blue arrows (Figure 4B). The current, when large enough, can flow for several myocytes (from myocyte 1 to myocytes 2 and 3) as shown in the diagram. Some of the axial current (capacitive current) depolarizes them to 


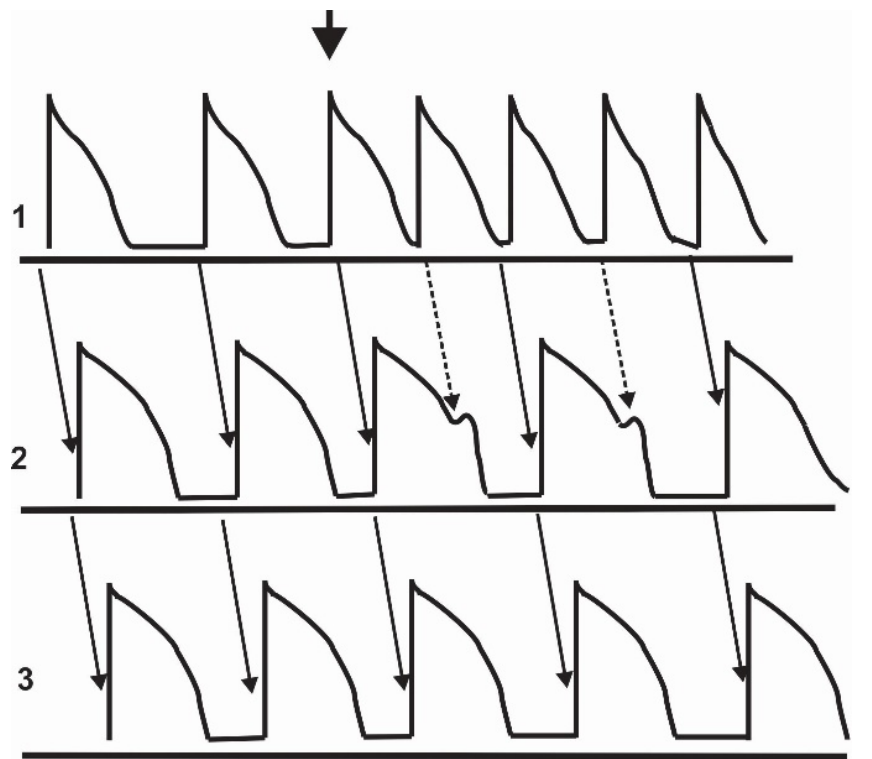

Figure 5. Phase 3 conduction block. Impulse conduction is diagrammed from His bundle (region 1) to more distal regions in the bundle branches (regions 2 and 3), indicated by the arrows. The cycle length is abruptly decreased at the large downward arrow causing 2:1 block of conduction. Block occurs because impulses from region 1 reach region 2 before it has sufficiently repolarized and is still in early phase 3 , generating only a small non conducted depolarization (dashed arrows).

the threshold potential so that they generate action potentials (Figure 4C). In extracellular space, current generated by the transmembrane current flow in myocytes 2 and 3, flows from these myocytes back to the excited myocyte, from positive to negative (blue arrows), completing the circuit. This is the current detected in electrogram recordings. The excited myocyte (1) generating the current in Figure 4B is the "source", the unexcited myocytes(s) (2 and 3) are the "sink." The action potential has conducted from myocyte 1 to myocytes 2 and 3 in Figure 4C (myocyte 1 has repolarized to reestablish its resting membrane potential of $-90 \mathrm{mV}$ ). Once threshold is reached and an action potential is generated in myocytes 2 and 3 , the newly excited cells switch from being a sink to being a source, generating axial current to depolarize downstream cells, perpetuating the process of action potential (impulse) propagation (Figure 4C).

Conduction properties depend to a large extent, on the properties of the ionic current generated by the source which is $I_{\mathrm{Na}}$ during phase 0 . A reduction of this current leads to a slowing of conduction and eventually conduction block. The main cause of such a reduction in $I_{\mathrm{Na}+}$ is based on the voltage dependence of inactivation and reactivation of the $\mathrm{Na}^{+}$channel. As described above (Figure $3 \mathrm{~A}$ ), the channel inactivates (closes), during phase 0 depolarization and then inactivation is removed during phase 3 repolarization. The myocyte cannot be re excited until phase 3 is almost finished. The time to repolarization determines the effective and relative refractory periods. Inactivation of the $\mathrm{Na}^{+}$channel, that occurs during Phase 0 of the action potential because of the decrease in membrane voltage, also occurs when there is a steady state reduction (becomes less negative) of the resting membrane potential, a reduction during phase 4. In Figure $3 B$ the level of the membrane potential during phase 4 is reduced. As a result voltage dependent inactivation of a fraction of $\mathrm{Na}^{+}$channels occurs, and inward $I_{\mathrm{Na}+}$ is reduced as is indicated by the slowing of phase 0 and reduction of total action potential amplitude (below). Conduction in these regions is very slow because of the reduced axial currents diagrammed in Figure 4D. The decreased rate and amplitude of phase 0 (red action potential), depolarizes the membrane potential only to $+5 \mathrm{mV}$ in myocyte 1 , reducing the amount of source current (blue arrows). This in turn decreases the rapidity at which cells in the pathway of propagation are depolarized to threshold potential, and the distance in front of the propagating action potential that is depolarized (axial current flows only to myocyte 2), slowing conduction. At these low levels of membrane potential, an inward $\mathrm{Ca}^{2+}$ current $\left(\mathrm{I}_{\mathrm{Ca}_{2+}}\right)$ that triggers contraction (not shown), also helps support conduction. Further reduction of the phase 4 resting potential (Figure 3C) inactivates a sufficiently high fraction of $\mathrm{Na}^{+}$channels to reduce $\mathrm{I}_{\mathrm{Na}+}$ to a level where action potentials and conduction cannot occur (Figure 4D).

One additional important property of Purkinje myocytes with reduced phase 4 membrane potentials must be recognized to understand the mechanism of block in the diseased His Purkinje system. Unlike normal Purkinje myocytes, the inactivated inward $\mathrm{I}_{\mathrm{Na}+}$ that supports conduction during phase 0 , does not completely recover when phase 3 repolarization returns membrane potential to phase 4 . Partial and 


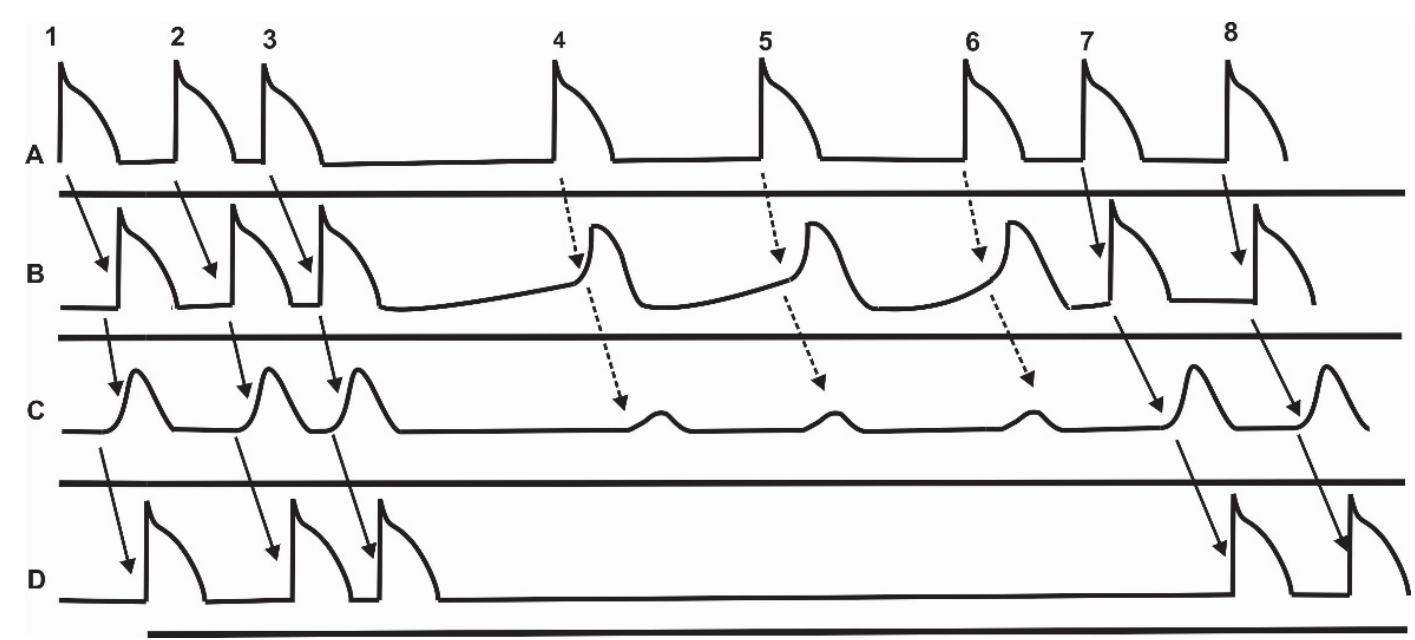

Figure 6. Mechanism for phase 4 block. Action potentials are shown in four contiguous regions in the His Purkinje system (A-D), with A representing the proximal His bundle. Propagation from one region to the next is indicated by the arrows (see text for description).

complete inexcitability (the relative and effective refractory periods) persist well into phase 4, called post repolarization refractoriness. Furthermore, as rate increases, rather than refractory periods of Purkinje myocytes shortening as they normally do (Purkinje myocyte repolarization accelerates and action potential duration shortens as cycle length decreases), the time for recovery gets longer much like the increase in $\mathrm{AV}$ nodal refractory periods with rate.

\section{What Is Phase 3 Conduction Block?}

Phase 3 block, which is physiological or functional block, can occur in the normal His Purkinje system after a conducted atrial premature depolarization or at the beginning of a supraventricular tachycardia. If a supraventricular impulse arrives in the His bundle or bundle branches while action potentials are in early phase 3 , it will block because $I_{\mathrm{Na}+}$ is not sufficiently recovered to allow impulse conduction (effective refractory period). Phase 3 block is facilitated if the duration of phase 3 is prolonged. This can occur after a sequence of a short-long preceding cycle length since the time for repolarization is directly related to the previous cycle length (see above). The duration of phase 3 is also prolonged in Purkinje myocytes in certain pathological situations, for example, in the conducting system in healed myocardial infarction or chronic heart failure. Although phase 3 block may persist for a number of impulses, it can also eventually disappear despite a persistent rapid rate since repolarization in the His Purkinje system is accelerated at rapid rates.

Figure 5 diagrams the mechanism for phase 3 block. Purkinje myocyte conduction is indicated from top to bottom by the slanted arrows, 1 representing the His bundle with shortest action potential duration and 2 and 3 representing more distal bundle branch with longer action potential durations. Activation cycle length is abruptly shortened at the large downward pointing black arrow leading to block at site 2 where the impulse from site 1 arrives during phase 3 , before recovery of excitability (dashed arrows). Block is facilitated when action potential duration at site 2 does not shorten normally at the decreased cycle length.

\section{What Is Phase 4 Conduction Block?}

The term "phase 4 block" indicates that conduction of an impulse arriving in the His Purkinje system during phase 4 , blocks as compared to block during phase 3 described above. It can also be thought of as bradycardia dependent block since it is initiated by a long cycle length or period of bradycardia although it might persist in the absence of bradycardia. $I_{\mathrm{Na}+}$ during an action potential initiated during phase 4 in the normal His Purkinje system, supports rapid conduction because of the high level of membrane 


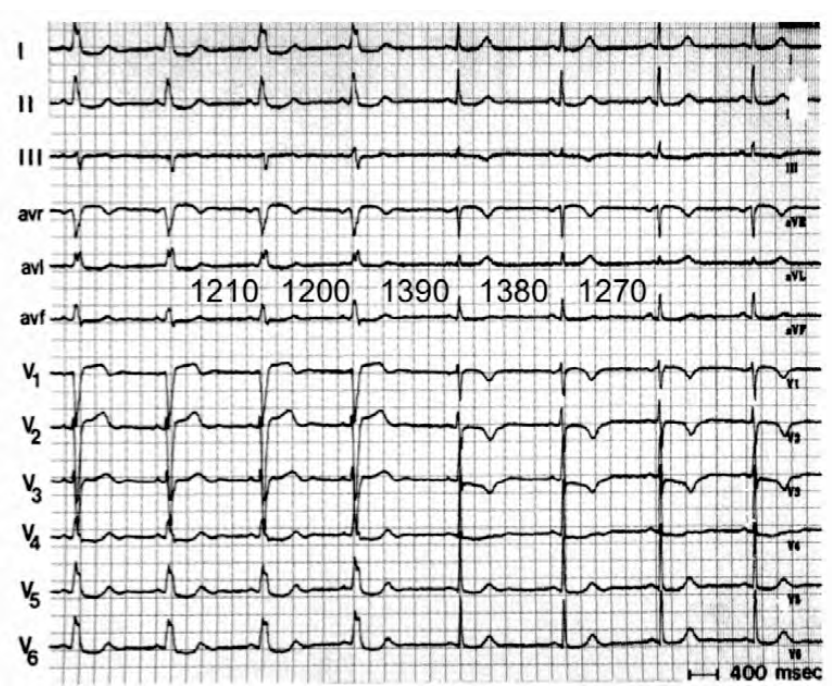

Figure 7. 12 lead ECG showing acceleration dependent LBBB which occurs at cycle lengths less than 1270 msec.

potential, so for conduction to block, $\mathrm{I}_{\mathrm{Na}}{ }^{+}$must be reduced. How does this occur?

The membrane potential during phase 4 is not always steady as depicted in Figure 2A. Purkinje myocytes are latent pacemakers and under special conditions express a pacemaker potential during phase 4 diagrammed in Figure 2B. Membrane potential during phase 4 decreases (becomes less negative) spontaneously (spontaneous phase 4 depolarization) because of an inward directed pacemaker current, until it reaches the threshold potential at which the $\mathrm{Na}^{+}$channels open to cause phase 0 and an action potential. This is the process of automaticity. Even though the pacemaker current and tendency for spontaneous phase 4 depolarization is normally present in Purkinje myocytes, it is usually suppressed at normal sinus rates, a phenomenon called "overdrive suppression" caused by activity of the $\mathrm{Na}^{+}-\mathrm{K}^{+}$pump in the sarcolemma which generates an outward membrane current that opposes phase 4 spontaneous depolarization. The pacemaker current can express itself if the over driving supraventricular impulses are slowed or inhibited. During long cycle lengths or in the absence of impulses entering the conducting system, inhibition of the pacemaker current is reduced, exposing spontaneous phase 4 depolarization, generating an automatic action potential (Figure 2B), that propagates through the conducting system leading to escape beats or idioventricular rhythm. In the diseased conducting system with a reduced level of membrane potential, the propensity for spontaneous diastolic depolarization is enhanced. Based on the relationship of membrane potential during phase 4 to the magnitude of phase 0 and to the conduction of the impulse described in Figures 3 and 4, it is apparent that the action potentials generated by spontaneous phase 4 depolarization, conduct more slowly because $\mathrm{I}_{\mathrm{Na}+}$ is partly inactivated at the reduced level of membrane potential at which the spontaneous action potentials occur. If the focus of spontaneous phase 4 depolarization and automaticity is small, it is unlikely that the slowly conducting action potentials generated in the focus will have any noticeable influence on activation of the rest of the His Purkinje system since once the impulse leaves the focus it will excite Purkinje myocytes with normal action potentials. However, if there is disease in a significant region characterized by reduced membrane potentials (Figure 3B), conduction block of impulses arising at the end of phase 4 depolarization can occur to cause bundle branch block or paroxysmal AV block. Figure 6 shows a model of phase 4 block caused by spontaneous phase 4 depolarization. Regions $A$ and $B$ are relatively normal although $B$ abuts an abnormal region $C$ which has a reduced membrane potential, reduced amplitude and slowed phase 0 due to partial $I_{\mathrm{Na}+}$ inactivation. Region D is distal to the abnormal region and has normal action potentials. During sinus rhythm (action potentials 1 and 2) conduction is maintained through the diseased region, indicated by the black arrows. As described above, phase 4 block can occur after a long cycle length. In the Shenasa case report [1], the long cycle is a result of a supraventricular premature impulse that resets the dominant sinus pacemaker. (Recall that a premature impulse resets a pacemaker by conducting into the pacemaker site and depolarizing the pacemaker cells prior to initiation of an impulse thereby causing a pause until the next spontaneous impulse is initiated). In the model of phase 4 block shown in Figure 6, action potential 3 in A represents a premature impulse which successfully propagates through the conducting system, from A to D (arrows). It is followed by a long cycle caused by resetting of the dominant pacemaker. That allows spontaneous phase 4 depolarization to occur in action potentials 


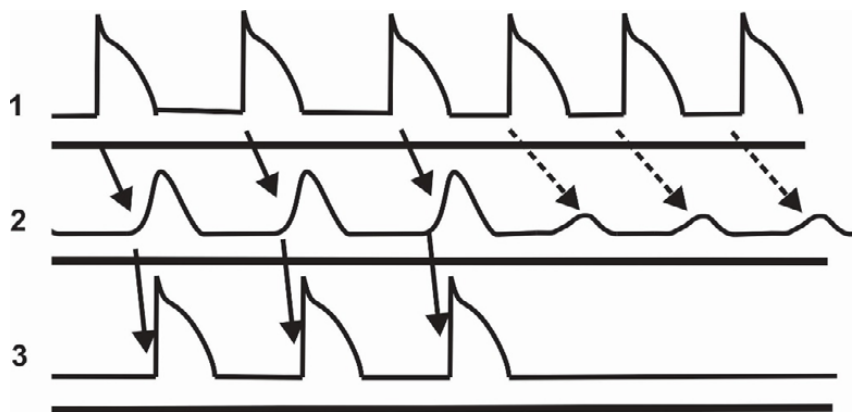

Figure 8. Mechanism for acceleration dependent block. Action potentials are shown in 3 contiguous regions in the His Purkinje system (1, 2, and 3) with region 2 having reduced action potentials because of disease. Conduction from 1 to 3 indicated by black arrows. A small decrease in cycle length causes block in region 2 (dashed arrows)

in $\mathrm{B}$. The impulse that arises in A after the long cycle arrives in region $B$ during phase 4 depolarization (impulse 4). Action potential 4 that occurs when region $B$ is excited has a reduced phase $0\left(I_{\mathrm{Na}+}\right)$ and because of the reduced axial current (see Figure 4D) is unable to excite the cells in region $C$ (dashed arrow), which because of their reduced membrane potentials are also more difficult to excite. This leads to block of conduction (dashed arrow) with failure of propagation to region $D$, either AV block if in the His bundle or bundle branch block if more distal. Phase 4 block may then persist for a variable period of time. Once spontaneous phase 4 depolarization occurs (after action potential 3), the rate of spontaneous phase 4 depolarization increases for subsequent action potentials (action potentials 4, 5, and 6) because of the decreased influence of the $\mathrm{Na}^{+}-\mathrm{K}^{+}$pump. In Figure 6, action potentials 5 and 6 from region $A$, also arrive in region $B$ after significant spontaneous phase 4 depolarization (dashed arrows), and therefore cannot excite region $\mathrm{C}$, leading to persistence of block.

Persistent block is favored if the input to region $B$ occurs at long cycles allowing spontaneous phase 4 depolarization. However this is not always the case. Sometimes supraventricular cycle length may shorten because of enhanced sympathetic activity from the baroreceptors or decreased coronary perfusion, both of which also enhance spontaneous phase 4 depolarization and decrease overdrive suppression, so phase 4 block might continue.

Restoration of conduction requires suppression

Wit, A.L. et al. of spontaneous phase 4 depolarization. This can be caused by a premature supraventricular or ventricular impulse that activates the cells at a short cycle length as shown in the Shenasa case [1]. In Figure 6, action potential 7 in region $A$ occurs prematurely and as a result activates region $B$ before spontaneous phase 4 depolarization reduces the membrane potential. Therefore, action potential 7 in region $B$ has a larger $\mathrm{I}_{\mathrm{Na}+}$ and a faster and larger phase 0 and is able to excite region $C$ (arrow), leading to resumption of conduction to the distal region (D). Action potential 8 in region $A$ is likewise able to conduct because spontaneous phase 4 depolarization is now suppressed. Other factors that suppress spontaneous phase 4 depolarization can also improve conduction. For example, in experimental studies, low doses of procainamide that suppress spontaneous phase 4 depolarization, increased phase 0 even though this drug in higher doses can decrease $\mathrm{I}_{\mathrm{Na}+}$ [4].

\section{What Is Acceleration-Dependent Block? How Does It Compare to Phase 3 and Phase 4 Block?}

Acceleration dependent block occurs after a modest increase in heart rate. Figure 7 shows ECG recordings with normal QRS complexes at cycle lengths of 1390 to 1270 and acceleration induced LBBB at cycle lengths less than 1270 . Although heart rate is still slow, it is the small decrease in cycle length that caused block in contrast to phase 3 block which occurs at short cycle lengths. Since Purkinje myocyte action potential duration is not more than about 500 msec even at long cycle lengths, block in Figure 7 cannot be occurring during phase 3 but must be occurring during phase 4.

As described in Figures 3B and $4 D, I_{\mathrm{Na}+}$ is reduced and conduction is slow when resting membrane potential (phase 4) is reduced. Refractory periods also last beyond full repolarization (post repolarization refractoriness) reducing excitability. Acceleration dependent block occurs in disease His Purkinje system with reduced resting membrane potentials. The effects of cycle length on conduction in a region of the diseased conducting system with low membrane potentials is diagrammed in Figure 8.

Impulses from region 1 which has normal action potentials, representing the proximal His bundle, 
propagate into a diseased region 2 with reduced membrane potentials in the more distal His bundle or bundle branches (region 2). Region 3 is distal to the diseased region. At the cycle length of the first three action potentials, conduction occurs through region 2 to excite the more normal distal conducting system (region 3) (arrows). However at the shorter cycle length of the next 3 action potentials, propagation blocks (dashed arrows) because the post repolarization refractoriness of diseased cells extends well into phase 4. This would lead to acceleration dependent AV block in the distal His bundle to cause complete AV block or more distally to cause bundle branch block.

\section{Summary}

Conduction block in the His Purkinje system results from a reduction in $\mathrm{Na}^{+}$current during phase 0 . The strength of this current to support conduction is dependent on the level of the membrane potential at which phase 0 arises. Phase 3 block occurs when action potentials enter a region that has not yet fully repolarized (phase 3 ), reducing $I_{\mathrm{Na}+}$. Phase 4 block occurs when spontaneous phase 4 depolarization reduces membrane potential, reducing $I_{\mathrm{Na}+}$ of action potentials propagating into a region of spontaneous phase 4 depolarization. Block can also occur during phase 4 in diseased regions with low membrane potentials during modest decreases in cycle length (acceleration dependent block) because of the post repolarization refractoriness at reduced membrane potentials.

\section{Conflict of Interest}

The authors have no conflict of interest relevant to this publication.

\section{Comment on this Article or Ask a Question}

\section{References}

1. Shenasa M. Paroxysmal atrioventricular block. Arrhythmia Grand Rounds. 2015;1:????. DOI: 10.12945/j.agr.2015.0033-15

2. Lee S, Wellens HJJ, Josephson ME. Paroxysmal atrioventricular block. Heart Rhythm. 2009;6:1229-1234. DOI: 10.1016/j. hrthm.2009.04.001
3. Hodgkin, AL. The conduction of the nervous impulse. Springfield, IL: Charles C Thomas; 1964.

4. Rosen, MR, Gelband, H, Hoffman, BF. Canine electrocardiographic changes induced by procainamide. Circulation. 1972;46:528536. DOI: 10.1161/01.CIR.46.3.528
Cite this article as: Wit $A L$, Josephson ME. Basic foundations of clinical electrophysiology. Electrophysiological mechanism of phase 4 conduction block in the his Purkinje system: a comparison with phase 3 block. Arrhythmia Grand Rounds 2015;1(2):79-86. DOI: http:// dx.doi.org/10.12945/j.agr.2015.033-15 